\title{
A new method for determining atmospheric pressure coefficient using fast Fourier transform for muons in the GRAPES-3 experiment
}

\author{
P.K. Mohanty ${ }^{* a, b}$, H.M. Antiaa ${ }^{a, b}$, K.P. Arunbabu ${ }^{a, b}$, S.R. Dugad ${ }^{a, b}$, S.K. Gupta ${ }^{a, b}$, B. \\ Hariharan $^{a, b}$, Y. Hayashi ${ }^{d, b}$, P. Jagadeesan ${ }^{a, b}$, A. Jain ${ }^{a, b}$, S. Kawakami ${ }^{d, b}, \mathbf{H}$. \\ Kojima $^{f, b}$, S.D. Morris ${ }^{a, b}$, P.K. Nayak ${ }^{a, b}$, T. Nonaka ${ }^{d, b}$, A. Oshima ${ }^{e, b}$, B.S. Rao ${ }^{a, b}$, S. \\ Shibata $^{e, b}$, P. Subramanian ${ }^{c, b}$, K. Tanaka ${ }^{g, b}$ \\ ${ }^{a}$ Tata Institute of Fundamental Research, Mumbai 400 005, India. \\ ${ }^{b}$ The GRAPES-3 Experiment, Cosmic Ray Laboratory, Raj Bhavan, 643001 Ooty, India. \\ ${ }^{c}$ Indian Institute of Science Education and Research, Pune 411 021, India. \\ ${ }^{d}$ Graduate School of Sciences, Osaka City University, Osaka 558-8585, Japan. \\ ${ }^{e}$ College of Engineering, Chubu University, Kasugai, Aichi 487-8501, Japan. \\ ${ }^{f}$ Faculty of Engineering, Aichi Institute of Technology, Toyota City, Aichi 470-0392, Japan. \\ ${ }^{g}$ Graduate School of Information Sciences, Hiroshima City University, Hiroshima 731-3194, \\ Japan. \\ Email: pkm@tifr.res.in
}

\begin{abstract}
A large area $\left(560 \mathrm{~m}^{2}\right)$ tracking muon detector operating in the GRAPES-3 experiment at Ooty in India has been recording cosmic ray muons at a rate of $1.7 \times 10^{8} \mathrm{~h}^{-1}$ since 2000 . The high statistics data have enabled sensitive measurements of several solar phenomena to be made including the solar and sidereal anisotropy and Forbush decreases associated with coronal mass ejections. Prior to studying of any of these phenomena, an important task is to correct the variation in measured muon rates due to atmospheric pressure. Unfortunately, the pressure coefficient usually deduced from the observed data is not very reliable due to the presence of various solar phenomena listed above. Here, we present an alternative method which avoids complications arising from solar effects. Since the pressure at Ooty displays a $12 \mathrm{~h}$ periodicity, using which we could separate its contribution from other effects in the muon data though a power spectrum analysis. The method yielded a clear dependence of muon rate on pressure providing an accurate estimate of the pressure coefficient almost independent of the solar modulation effects.
\end{abstract}

The 34th International Cosmic Ray Conference,

30 July- 6 August, 2015

The Hague, The Netherlands

\footnotetext{
*Speaker.
} 


\section{Introduction}

The GRAPES-3 is an extensive air shower experiment operating at Ooty in South India $\left(11.4^{\circ} \mathrm{N}\right.$ latitude, $76.7^{\circ} \mathrm{E}$ longitude and $2200 \mathrm{~m}$ altitude) since 2000 . It comprises of an array of $\sim 400$ plastic scintillator detectors of $1 \mathrm{~m}^{2}$ area each, placed in hexagonal rings with $8 \mathrm{~m}$ inter-detector spacing and a large area $\left(560 \mathrm{~m}^{2}\right)$ tracking muon detector $[1,2]$. The muon detector consists of 16 separate modules each of area $35 \mathrm{~m}^{2}$. The basic elements of each module are the proportional counter (PRC) tubes $(6 \mathrm{~m}$ in length and $10 \mathrm{~cm} \times 10 \mathrm{~cm}$ in cross section) which have been arranged in four layers in two orthogonal planes. This configuration is used to determine the direction of the muons into $13 \times 13$ (169) solid angle bins in the field of view of the detector [3]. Concrete blocks of $550 \mathrm{~g} \mathrm{~cm}^{-2}$ thickness have been used as absorber to shield the electromagnetic components, while providing $1 \mathrm{GeV}$ threshold for vertically incident muons. Each module records nearly 3000 muons per second, thus allowing to make sensitive measurements on various solar phenomena including Forbush decreases associated with coronal mass ejections, diurnal and sidereal anisotropies $[3,4,5,6]$.

The atmospheric pressure produces a significant variation in the muon rates apart from the variations in the primary cosmic rays associated to various solar phenomena. At Ooty, the pressure data exhibits a regular $12 \mathrm{~h}$ periodicity with amplitude of about $1 \mathrm{hPa}$ in addition to the other seasonal variations. The $12 \mathrm{~h}$ period is significant and dominant primarily in the equatorial regions like Ooty. The predominance of a harmonic amplitude at a given geographical location depends on several factors such as water vapor heating, diurnal temperature, landmasses and wind direction. A detail discussion of this topic can be found in [7] and the references therein.

The atmospheric pressure and the muon flux follow an anti-correlation which can be fairly described by a linear relation to first order approximation. While the pressure correction of data is relatively straight forward because of the above fact, however estimation of an reliable and accurate value of pressure coefficient is generally not simple because of a number of practical difficulties. A major difficulty arises due to the interference of the solar diurnal anisotropy. It is worth noting that the amplitude of the solar diurnal anisotropy can be comparable or even larger to that induced by pressure during high solar activity periods. Transient variations such as Forbush decreases or geomagnetic storms can adversely impact the measurement of pressure coefficient. Further there could be seasonal changes in pressure and irregular changes in cosmic ray intensity. To get a reliable value of pressure coefficient, the standard practice is to select periods of low solar activities or identify the periods of transient events and exclude them from the analysis [8]. Nevertheless, many decreases are difficult to localize, especially in the muon component, and even small long term variation may affect the result seriously. Therefore, it is generally difficult to get a reliable value for pressure coefficient by this conventional analysis method.

\section{The muon and pressure data sets}

In this analysis, we used data over a period of one year (1 January to 31 December 2006). We used muon rates averaged over 4 min which was represented by $R_{4 \min }$. Various instrumental effects in the muon data such as gaps, spikes, jumps and fluctuations were identified through visual inspections and these periods were excluded from further analysis. Apart from the short lived variations, the rates also exhibit gradual variations which were caused mainly due to slow leaking 
of gas from PRC. These variations were modeled by fourth order polynomial fits and corrected [9]. Thereafter the data from the 16 modules were combined to improve the statistical accuracy and minimize the gaps. The pressure measurement in this period was carried out by using two independent digital barometers which provides data with resolution of $0.1 \mathrm{hPa}$ of each at intervals of 1 min which were combined to obtain 4 min bins referred as $\mathrm{P}_{4 \min }$. An effective self-consistent calibration method discussed elsewhere [5] was used to obtain an uninterrupted data set for the entire period despite of gaps occurred in the individual instruments.

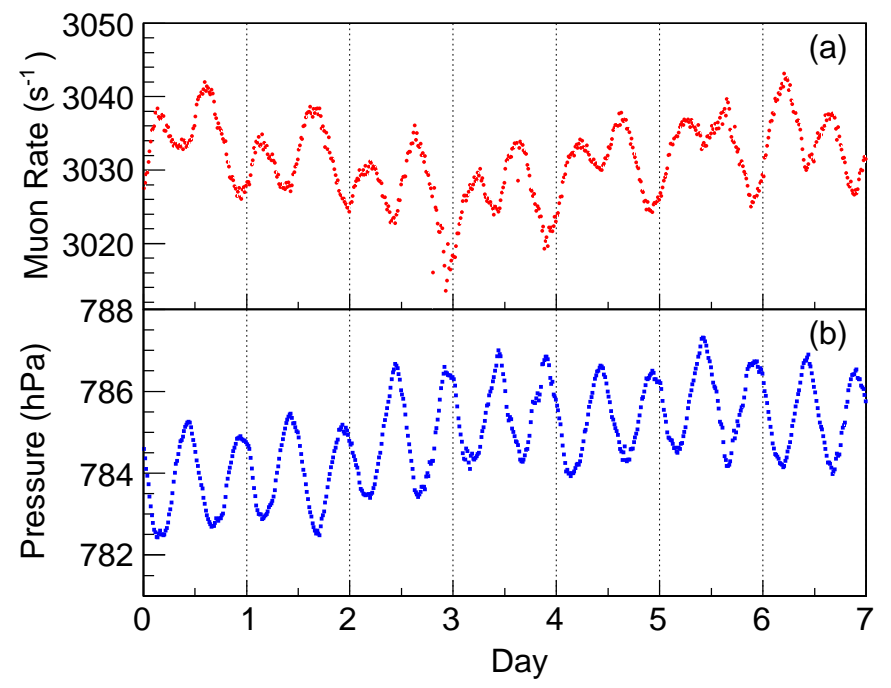

Figure 1: Variation of (a) mean muon rate, (b) pressure (hPa) for the week 1-7 March 2006.

The variation of muon rate and the atmospheric pressure over a period of one week interval from 1 to 7 March 2006 is shown in Fig. 1. Each data point in the plot corresponds to an interval of $16 \mathrm{~min}$ which was obtained after re-binning the 4 min intervals of data to reduce the statistical fluctuation. An anti-correlation between the muon rate and the atmospheric pressure may be observed along with a dominant $12 \mathrm{~h}$ periodicity in both data sets. However, a careful examination of the plots in Fig. 1a and Fig. 1b showed that while the pressure data displayed an almost sinusoidal profile riding on a slowly rising ambient pressure, the muon rate exhibited a complex profile. This complexity primarily arose as a result of the presence of the solar diurnal anisotropy that modulated the muon rate with a $24 \mathrm{~h}$ period and the interference of these two periodicities ( $12 \mathrm{~h}$ and $24 \mathrm{~h}$ ) resulted in the observed muon rate profile.

\section{Conventional determination of pressure coefficient}

To appreciate the superiority of the fast Fourier transform (FFT) method which is discussed later in $\S 4$, the conventional method and its complexity was demonstrated below. The dependence of $\mathrm{R}_{4 \min }$ on $\mathrm{P}_{4 \min }$ obtained using the data of entire 2006 is shown in Fig. 2. Clearly, the dependence of the muon rate on pressure shows a complex profile than a simple linear trend expected if it was purely due to the pressure contribution. A linear least square minimization to the data exhibited a poor fit shown as dashed line in Fig. 2. The poorness of the fit is also reflected in the large value of 


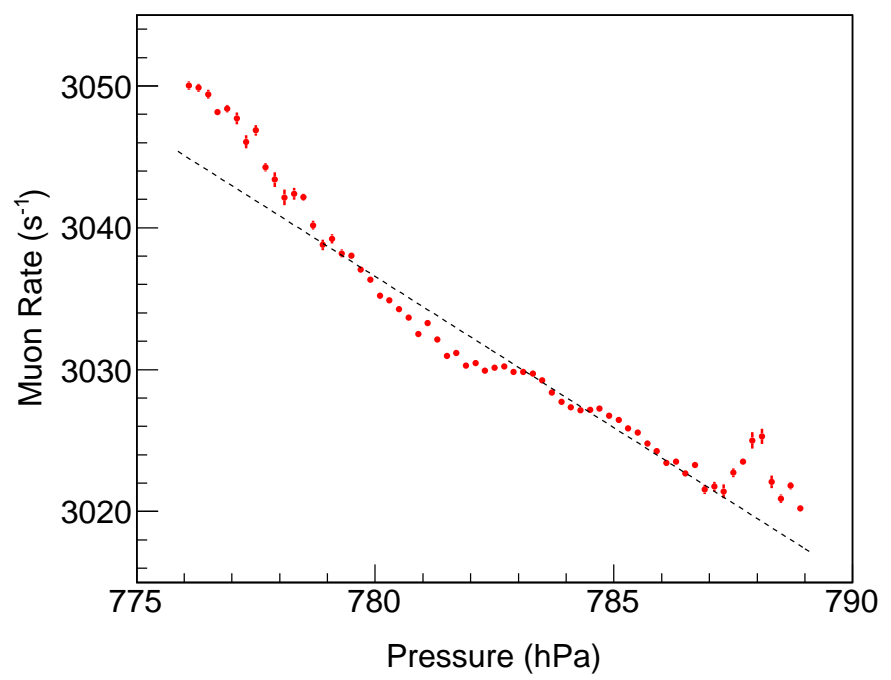

Figure 2: Variation of muon rate as a function of atmospheric pressure (hPa) with complete 2006 data. The dashed line represents a linear least square fit to the data. Slope of the fit $=0.07 \% / \mathrm{hPa}$ and normalized $\chi^{2}=81$ per dof.

$\chi^{2}$ per degree of freedom (dof) which is to be 81 here. The pressure coefficient $(\beta)$ value obtained from the fit is 2.13 counts $\mathrm{s}^{-1} / \mathrm{hPa}$ and a translated fractional value of $0.07 \% / \mathrm{hPa}$ for the mean value of 3028.09 counts $s^{-1}$.

\section{Determination of pressure coefficient using FFT method}

The pressure data at Ooty exhibited primarily a $12 \mathrm{~h}$ periodicity which can be seen in Fig. 1 . It was expected that the $12 \mathrm{~h}$ periodicity would also produce same periodic response in the muon rate due to the anti-correlation of the two observables. To examine this hypothesis, we performed frequency analysis on both the data sets using FFT technique,. For this purpose, an uninterrupted data set of $2^{17}$ time intervals of $\mathrm{R}_{4 \min }$ spanning $364.01 \mathrm{~d}$ from 1 January 2006 was used. Next, percent deviation of each $\mathrm{R}_{4 \min }$ from the mean value of entire considered period was calculated. The small fraction of gaps in the muon data were padded with zeros. Then FFT was performed on both the time series data of muon rate and pressure using a routine namely TVirtualFFT built in the data analysis software ROOT (http://root.cern.ch/root/html/TVirtualFFT.html).

The FFT spectrum of the muon rate and pressure data are shown in Fig. 3a and b respectively. The amplitude for frequencies above 8.5 cycles per day (cpd) are consistent with the expectation from the background. The spectrum of muon data showed large peaks at 1 and $2 \mathrm{cpd}$. The highest amplitude was observed at $2 \mathrm{cpd}$ followed by $1 \mathrm{cpd}$ and thereafter amplitudes become rapidly smaller. A similar profile was seen in the amplitude spectrum of the pressure data with the highest peak at $2 \mathrm{cpd}$. This analysis clearly established the dominance of $2 \mathrm{cpd}(12 \mathrm{~h}$ period $)$ in the pressure data at the GRAPES-3 experimental site. The peaks in the muon spectrum included the contribution of the pressure as well as that of the solar diurnal anisotropy. A comparison of pressure peak at $1 \mathrm{cpd}$ with the corresponding peak of muon data shows that the pressure has a minor contribution. However, the 2 cpd peaks are comparable in both data implying the primarily contribution of pres- 


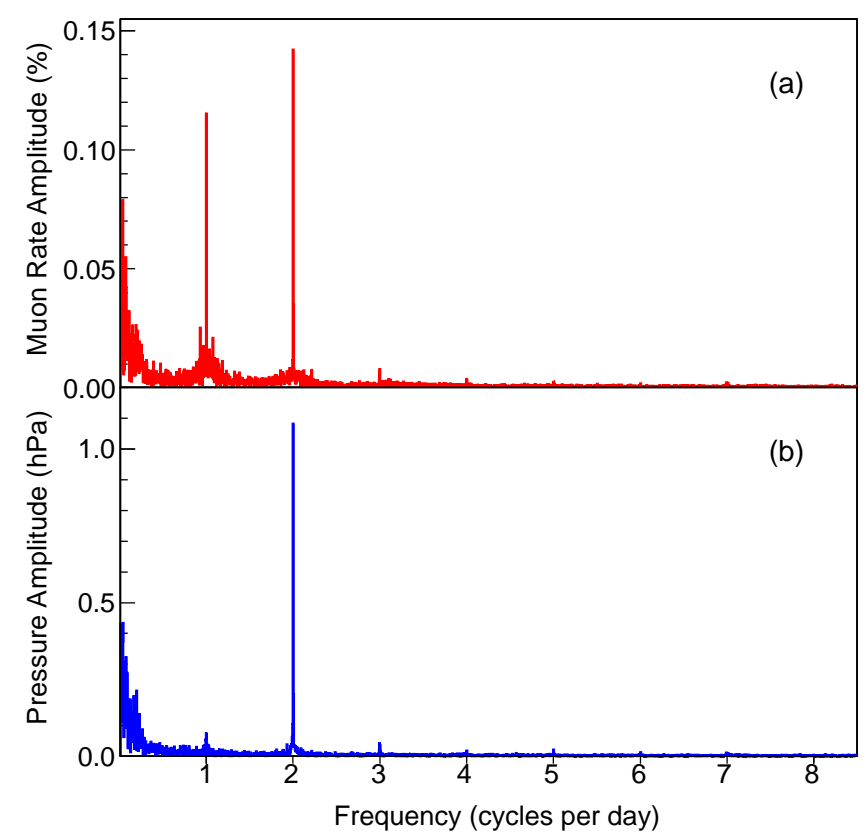

Figure 3: FFT spectrum of, (a) muon rate, (b) atmospheric pressure for $2^{17}$ of 4 min samples over $364.01 \mathrm{~d}$ in 2006.

sure effect. This unique feature of the data was exploited to segregate the non-atmospheric effects in the muon data to obtain a better estimate of $\beta$ as discussed below.

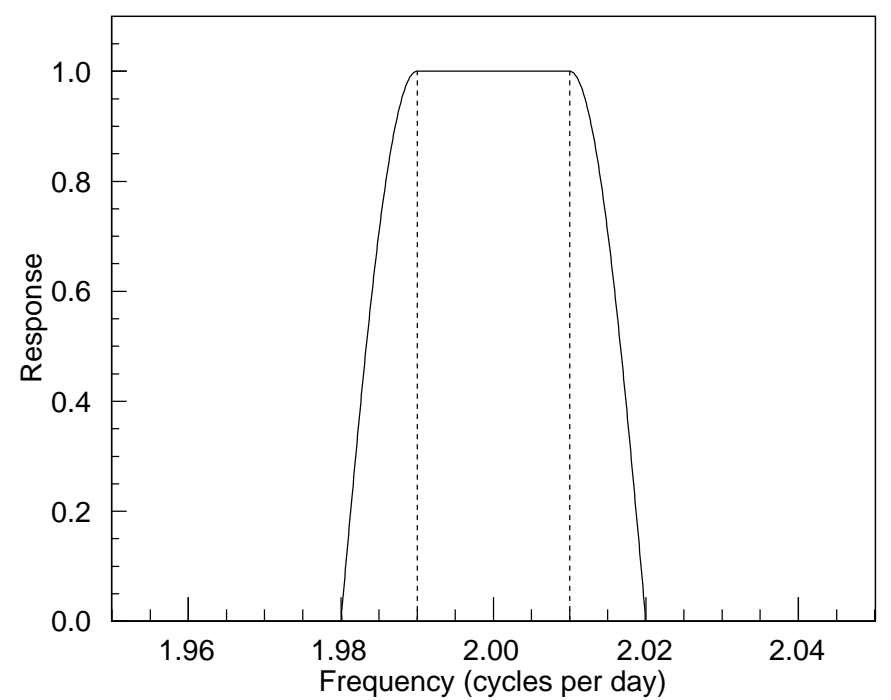

Figure 4: Filter used to select frequencies centered at $2 \mathrm{cpd}$ in muon and pressure data. The region of 100\% acceptance is enclosed by two dashed vertical lines.

A narrow pass filter $W(f)$ was designed to select the frequencies centered at $2 \mathrm{cpd}$ as described 
below,

$$
W(f)= \begin{cases}1, & \text { if }\left|f-f_{c}\right| \leq \Delta f \\ \sin \frac{\pi}{2} \frac{\left|f-f_{c}\right|}{\Delta f}, & \text { if } \Delta f<\left|f-f_{c}\right| \leq 2 \Delta f \\ 0, & \text { if }\left|f-f_{c}\right|>2 \Delta f\end{cases}
$$

Here $f_{c}$ represented the central frequency. In Fig. 4 the filter used in the present analysis constructed with $f_{c}=2 \mathrm{cpd}$ and $\Delta f=0.01$ is shown. The filter had $100 \%$ acceptance in the frequency range from 1.99 to $2.01 \mathrm{cpd}$ and it smoothly decreased to zero following a sine function in the range $1.99-1.98 \mathrm{cpd}$ and $2.01-2.02 \mathrm{cpd}$, respectively. The acceptance was zero outside of this range. Such a design choice was made to avoid possible ringing effects introduced by windows with sharp cutoff edges such as a rectangular window [10].

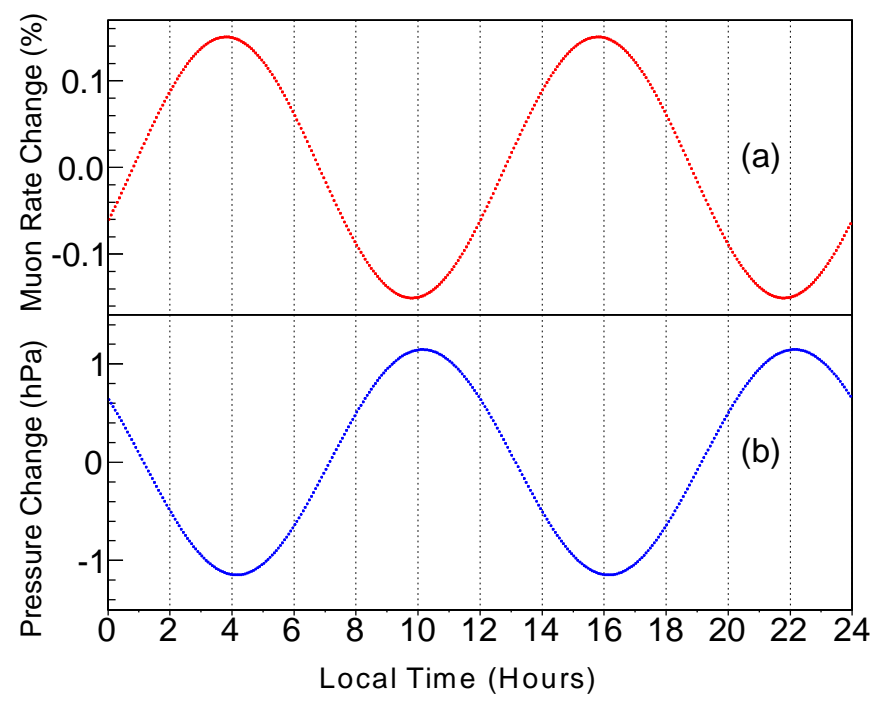

Figure 5: Time domain data after IFFT, folded modulo $24 \mathrm{~h}$ for, (a) muon rate, (b) pressure.

An inverse fast Fourier transform (IFFT) was performed on the filtered frequency spectrum to obtain the data in time domain. The resultant time series for entire 2006 was folded modulo $24 \mathrm{~h}$ as shown in Fig. 5a for muon and in Fig. $5 \mathrm{~b}$ for pressure. The pressure curve showed minima at 4 AM and $4 \mathrm{PM}$, while the maxima are at $10 \mathrm{AM}$ and $10 \mathrm{PM}$ local time. The peaks in the muon rate curve are almost anti-correlated with pressure with a phase difference of about $20 \mathrm{~min}$. This showed that the $12 \mathrm{~h}$ period $(2 \mathrm{cpd})$ in the muon rate was caused primarily by the pressure variation.

The IFFT data of muon rate was plotted against pressure as shown in Fig. 6. A linear fit to the data in Fig. 6 represented by a solid line displayed a nearly perfect fit. The slope of line which represents $\beta$ was found to be $-0.1284 \% / \mathrm{hPa}$.

The observed $R_{4 \min }$ data were corrected using value of $\beta=-0.1284 \% / \mathrm{hPa}$ as shown below,

$$
R_{c o r}=\frac{R_{o b s}}{(1+\beta \Delta P)}
$$

Where $\Delta P$ is the change from the mean pressure. 


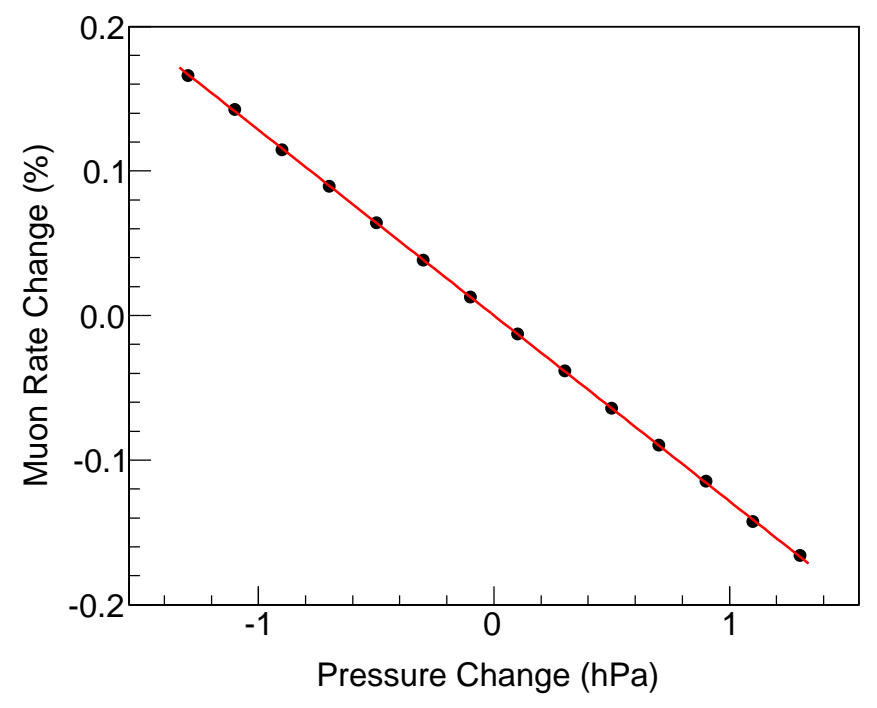

Figure 6: Variation in muon rate as a function of pressure relative their mean values.

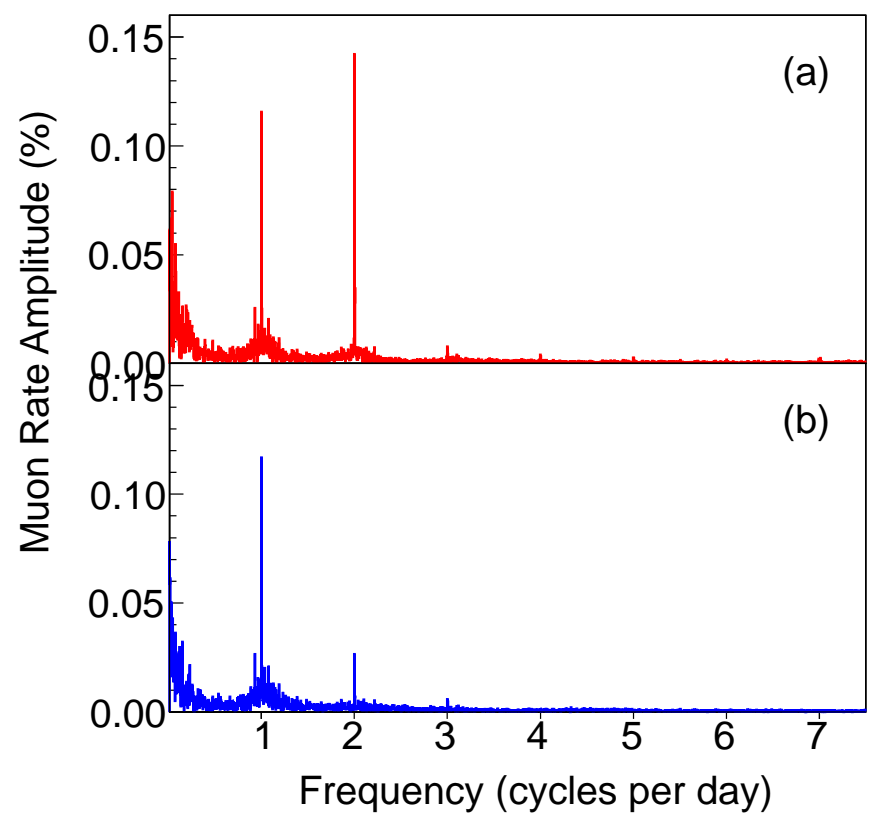

Figure 7: Frequency spectrum for muon data, (a) before pressure correction, (b) after pressure correction.

FFT was performed on the pressure corrected muon rates. The FFT spectrum before pressure correction is shown Fig. 7a and after the pressure correction is shown in Fig. 7b. As expected, the amplitude corresponding to the frequency of $1 \mathrm{cpd}$ remained almost unaffected after the correction, primarily because the corresponding pressure amplitude was very small. However, the effect on the frequency of $2 \mathrm{cpd}$ was dramatic since the pressure amplitude was dominant for this frequency. 
The residual amplitude after the correction shows the presence of a sizable amplitude of solar semidiurnal component ( $2 \mathrm{cpd}$ ). Another peak at $3 \mathrm{cpd}$ was also visible. Peaks at still higher frequencies could not be observed indicating that the higher frequency peaks in the uncorrected spectrum were caused by pressure effects.

\section{Summary}

Though, it is a well-known fact that the muon rate and pressure are anti-correlated, the interference of solar diurnal effects, Forbush decreases and seasonal variations had complicated the anti-correlation as observed in the data. To get an accurate estimate of the pressure coefficient in this situation, we devised a novel method using FFT technique. FFT analysis was performed on the muon rate and atmospheric pressure data and the amplitude spectra in frequency domain was obtained. The amplitude spectrum of the muon data showed peaks at 1,2,3 cpd, with the highest peak occurring at $2 \mathrm{cpd}$ followed by the peak at $1 \mathrm{cpd}$ and thereafter relatively smaller peaks at higher frequencies. Similar profile was seen in the amplitude spectrum of the pressure data with the highest peak being seen at $2 \mathrm{cpd}$. This analysis clearly showed the dominance of $2 \mathrm{cpd}(12 \mathrm{~h}$ period) in the pressure data at the GRAPES-3 experimental site. The spectral power centered at $2 \mathrm{cpd}$ for the pressure and the muon rate data was extracted by using a narrow band filter and IFFT performed to obtain the pressure and muon rate data in time domain. The new muon rate data were plotted against the pressure data and a perfect anti-correlation between the two was observed for the 2 cpd component. The value of the pressure coefficient $\beta=0.1284 \% \mathrm{hPa}^{-1}$ was obtained by using a linear fit from the regression plot. After the muon rate was corrected for variations in pressure, the amplitude of the diurnal component ( $1 \mathrm{cpd}$ or $24 \mathrm{~h}$ period) remained almost unaltered and the residual amplitude at $2 \mathrm{cpd}(12 \mathrm{~h}$ period) showed the presence of the semi-diurnal solar anisotropy.

\section{References}

[1] S. K. Gupta et al., Nucl. Instrum. Meth. A 540 (2005) 311.

[2] Y. Hayashi et al., Nucl. Instrum. Meth. A 545 (2005) 643.

[3] T. Nonaka et al., Phys. Rev. D 74 (2006) 052003.

[4] K. P. Arunbabu et al., Astron. Astrophys., 555 (2013) A139.

[5] P. K. Mohanty et al., Pramana - J. Phys. 81 (2013) 343.

[6] H. Kojima et al., Astroparticle Phys. 62 (2015) 21.

[7] A. Dai et al., J. Atmos. Sci. 56 (1999) 3874.

[8] C. P. Baker et al., "Proc. 23rd ICRC, Calgary 3 (1993) 753.

[9] S. K. Gupta, EPJ Web of Conferences 52 (2013) 04005.

[10] F. J. Harris, Proc. IEEE 66 (1978) 51. 